\title{
Genotype-specific habitat selection for oviposition sites in the cactophilic species Drosophila buzzatii
}

\author{
J. S. F. BARKER*, WILLIAM T. STARMER $†$ \& JAMES C. FOGLEMAN $\ddagger$ \\ Department of Animal Science, University of New England, Armidale, NSW 2351, Australia, $\dagger$ Department of Biology, \\ Syracuse University, Syracuse, NY 13244 and $\ddagger$ Department of Biology, University of Denver, Denver, CO 80208, U.S.A.
}

\begin{abstract}
Isofemale lines of the cactophilic species, Drosophila buzzatii, exhibit genetic variation for their oviposition response to cactus yeast species in the laboratory. In general, interactions between yeast species preclude the use of pairwise preferences as predictors of preferences in three-way choice experiments. Two isofemale lines with relatively high laboratory preference for ovipositing on the yeast Pichia cactophila (as opposed to Cryptococcus cereanus) and two isofemale lines with relatively low preference for $P$. cactophila were used in a series of field release experiments to determine if laboratory preferences were also realized under field conditions. The influence of yeast species on both settling behaviour (long-distance response) and oviposition preference (shortdistance response) were tested. The four lines were identical in their settling behaviour, preferring $P$. cactophila. The analysis of the oviposition preference tests showed significant line effects which correlated with the laboratory results. Thus a genetic component for oviposition preference under laboratory and field conditions was demonstrated and this strengthens the evidence for genotypespecific habitat selection in $D$. buzzatii. One low line, however, did not differ significantly from the two high lines under field conditions. A laboratory retest of this low line showed that the laboratory preference had not changed. The reason for the difference in the two situations is unknown but undoubtedly is attributable to uncontrolled variables under the field situation. Settling behaviour and oviposition response, in general, appear to be proximately linked to differences in the volatiles produced by the different yeast species.
\end{abstract}

Keywords: cactophilic yeasts, Drosophila buzzatii, Drosophila-yeast interactions, genetic variation, habitat selection, oviposition preference.

\section{Introduction}

Habitat selection can influence many aspects of the evolutionary ecology of populations or species (Rosenzweig, 1987; Orians, 1991). From an evolutionary genetic standpoint, one aspect of particular importance is the maintenance of genetic variation (Jaenike \& Holt, 1991). Theoretical studies (Maynard Smith, 1970; Taylor, 1976; Templeton \& Rothman, 1981; García-Dorado, 1986, 1987; Hedrick, 1990a, b) show that conditions for the selective maintenance of polymorphism are generally enhanced by genotypespecific habitat preferences, with the fitness of each genotype highest in its preferred habitat. Genetic variation can be maintained, however, at a locus affecting habitat preference, even when this locus does not affect

${ }^{*}$ Correspondence. fitnesses in the different habitats (Rausher, 1984; Diehl \& Bush, 1989).

Animals living in the same local region can exhibit several hierarchical stages of habitat selection. Success or failure in activities such as foraging, hiding, mating and oviposition can be influenced by differences in the general places that are suitable for these activities and also by differences in the patches within those places where the behaviour takes place. This concept of a spatial hierarchy in habitat selection extends to a temporal (suitability of places over time) as well as a genetic hierarchy. In the genetic hierarchy, different species can move to and occupy different habitats, while different genotypes of the same species can use different patches within a habitat. The feeding and breeding behaviour of host-specific insects exemplifies the higher levels of the genetic hierarchy. This is especially true for host-use patterns in those species of 
Drosophila where closely related taxa use different but closely related host plants (Heed, 1971; Fellows \& Heed, 1972). In these examples, the host plants represent a spatial hierarchy onto which the genetic hierarchy is overlaid.

The cactophilic Drosophila that use decaying tissue of cactus species as habitats represent an excellent system with which to study genotype-specific habitat selection. Most of the activities of these flies are carried out within the context of the host plants. Adults move to and settle on the host plants where they feed, mate and females oviposit. Eggs are laid in microhabitats that can differ from one another in terms of stage of decay, types and quantities of microorganisms present and physical conditions such as moisture content, $\mathrm{pH}$ and temperature. The oviposition choices made by females have a large potential influence on their progeny. This influence would augment habitat selection and future female oviposition choices if those choices are affected by the genotype of the female. Barker (1992) has shown that different genotypes (different isofemale lines) of Drosophila buzzatii, a cactophilic species which utilizes necrotic Opuntia (prickly pear) pads, show different preferences when given a simultaneous choice of five cactophilic yeast species as oviposition sites. Thus, direct evidence exists for genotype-specific habitat preferences in laboratory experiments. The heritabilities of individual female preferences for each yeast (proportion of eggs laid on yeast $i$ versus the combined total of the other four yeasts) were generally not significantly different from zero for two of the yeasts (Candida sonorensis and Clavispora opuntiae) but ranged up to about 10 per cent for the other three (Pichia cactophila, Candida mucilagina and Cryptococcus cereanus).

With five yeasts offered simultaneously, each of these estimates of the heritability of preferences for a particular yeast may well be affected by interactions among the other four yeasts that modify the observed preferences. The experimental test system, however, does simulate the multiple choice commonly available within rots in nature, as these rots contain one to five yeast species, with an average of about two to three (Barker et al., 1983, 1984). While simulating nature in that respect, the laboratory system is obviously standardized (all females exposed to the same five yeasts) and under a controlled environment, so that the genetic variation demonstrated in the laboratory may not be expressed in the field.

In this paper, we report on genetic variation among 90 isofemale lines of D. buzzatii for oviposition preferences for three yeast species, on experiments designed to evaluate the effects of interactions among yeast species on preferences and on the repeatability of laboratory preferences under field conditions.

\section{Materials and methods}

\section{Oviposition preferences of isofemale lines}

Experiments 1 to 4 used 10 isofemale lines from each of nine populations (Fig. 1) of $D$. buzzatii. The yeast species tested in the four experiments were $P$. cactophila (Pc, strain 77-168) and $\mathrm{Cl}$. opuntiae (Clo, strain 77-182) in experiment $1, P$. cactophila and $C r$. cereanus (Crc, strain 77-140B) in experiment $2, \mathrm{Cl}$. opuntiae and $\mathrm{Cr}$. cereanus in experiment 3 and all three in experiment 4 . The isofemale lines had been maintained in the laboratory on cactus medium (Starmer \& Barker, 1986) for three, four, 28 and nine generations, respectively, before initiation of each experiment and without exposure to cactophilic yeasts or bacteria.

All procedures for preparation of experimental flies, microorganism culture and setting up the oviposition test chambers were identical for the four experiments (with one exception for experiment 3: see below) and were generally the same as those described in detail by Barker (1992). Five replicate cultures (three pairs of parents per replicate) were set up for each isofemale

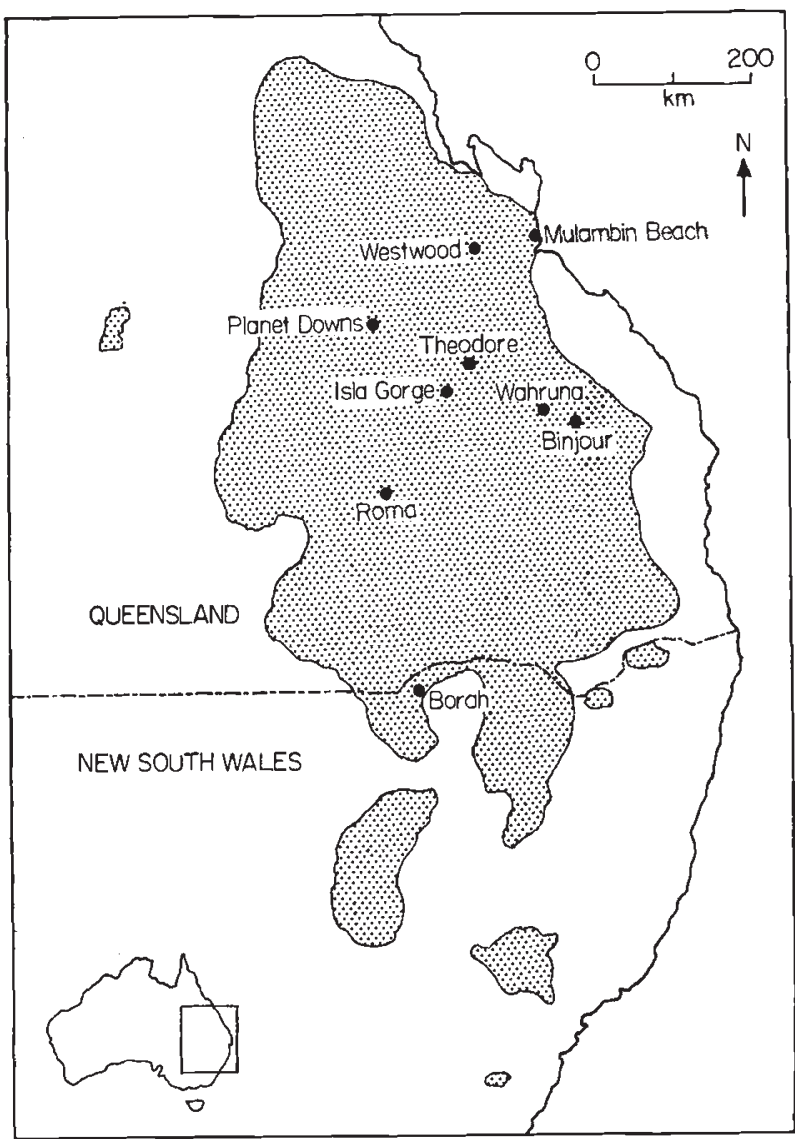

Fig. 1 Locations of the populations from which isofemale lines were collected. The hatched area shows the distribution of the main Opuntia infestations in 1920. 
line on autoclaved cactus medium seeded with live $S$. cerevisiae. Ten pairs of progeny from each replicate were stored on this same medium and transferred to fresh vials every other day until used in an experiment. The three yeast species used were chosen on the basis of the results of Barker (1992): P. cactophila and $C r$. cereanus (for which D. buzzatii showed heritable variation for oviposition preferences) and $\mathrm{Cl}$. opuntiae (for which no such variation was detected). A bacterial community (six different species isolated from natural rots) (Barker, 1992) was used with each yeast species to simulate the microorganism composition of rots in nature. Each yeast species, together with the mixed bacteria community, was presented to the flies as $48 \mathrm{~h}$ cultures growing on the surface of a cactus homogenate medium. For experiments 1, 2 and 4, the medium was 10 per cent Opuntia stricta homogenate, 1.5 per cent agar but for experiment 3,30 per cent $O$. stricta homogenate was used. Experiment 3 was carried out after the field experiment and when we had demonstrated that the yeast species grew better on the more concentrated homogenate.

Oviposition chambers were $9 \mathrm{~cm}$ Petri dishes with a cotton-stoppered hole in the lid (for addition of flies) and a filter paper in the base moistened with $1 \mathrm{ml}$ sterile water. Discs $1.6 \mathrm{~cm}$ diameter were cut from the microbial culture slabs and placed around the periphery of each chamber. For experiments 1,2 and 3, two discs of each of the two yeast species were equally spaced opposite each other so that the yeast species alternated. For experiment 4, two discs of each of the three yeast species were similarly arranged.

The five females (9-day-old) to be added to each chamber were taken at random from the stored flies of each replicate under light $\mathrm{CO}_{2}$ anaesthesia and placed in vials containing $5 \mathrm{ml}$ of 1.5 per cent agar. Thus the replicate sets of experimental females within isofemale lines did not share a common environment, either as larvae or as adults. Between 2.15 and 3.15 p.m., the females were added to the oviposition preference chambers and the chambers then arranged in random order and placed in a dark incubator at $25^{\circ} \mathrm{C}, 75$ per cent r.h. by 3.30 p.m. At 8.00 p.m. the following day, chambers were put in a freezer at $-15^{\circ} \mathrm{C}$ for $30 \mathrm{~min}$ and then in a cold room at $0^{\circ} \mathrm{C}$ to prevent further embryo development. The eggs on each disc were counted over the next few days. As $D$. buzzatii females show a major peak of oviposition in the late afternoon/ early evening, the test period includes 2 days of egg laying.

Data on oviposition preferences were analysed by ANOVA of the proportions of eggs laid on $P$. cactophila in experiments 1 and 2, on Cr. cereanus in experiment 3 and on each of the three yeasts in experiment 4 .
Total eggs laid in each replicate chamber was also analysed. The $F_{\max }$ test (Sokal \& Rohlf, 1981, p. 403) showed no heterogeneity of variances for populations or for lines although there were a few cases of deviations from normality. However, ANOVA following Box-Cox-Bartlett transformation gave the same results as for the untransformed data, so only the latter are presented.

\section{Field experiments}

The field experiments tested preferences for P. cactophila and $\mathrm{Cr}$. cereanus only and used four lines selected from those tested in the isofemale line experiments. A suitable oviposition surface was prepared by homogenizing Opuntia stricta cladodes, autoclaving and adding 1 per cent $(\mathrm{w} / \mathrm{v})$ Bacto agar, boiling, cooling and pouring into sterile $10 \times 60 \mathrm{~mm}$ Petri dishes. The solid cactus-agar surface was inoculated and spread with a $0.5 \mathrm{ml}$ suspension $\left(10^{6}-10^{7}\right.$ cells $\left./ \mathrm{ml}\right)$ of the bacterial community previously mentioned and one of two yeasts: $P$. cactophila or Cr. cereanus. The plates were incubated at $28^{\circ} \mathrm{C}$ for $48 \mathrm{~h}$ before use.

Two types of field tests were conducted. In one case, two types of oviposition-choice plates, one inoculated with Pc and one inoculated with Crc, were placed together in the same plastic container (two plates/ container), while in the other, the two types were placed in separate containers (one plate/container). Plastic containers were round $(11 \mathrm{~cm}$ in height, $17 \mathrm{~cm}$ in diameter) and covered by a lid with a central metal grid $(7.5 \mathrm{~cm}$ in diameter $)$ that would allow access to flies. The plastic containers with yeast plates inside were placed according to a systematic pattern (12 spots; Fig. 2) at each field locality. Localities with one plate/container had six containers with Pc-inoculated plates and six containers with Crc-inoculated plates systematically interspersed (Fig. 2). Localities with two plates/container had 12 containers each with a Pc and a Crc-inoculated plate. At each container placement spot, a wooden stake was driven into the ground and a paper plate stapled to the stake so that the container was shielded from direct sunlight and rainfall. Field localities were in the vicinity of Armidale, N.S.W., Australia, where there are no naturally-occurring stands of Opuntia. Four sectors were designated, each one for release of one of the four lines. Within each sector, five or six release sites were chosen with the minimum distance between them at least $2.6 \mathrm{~km}$ whereas the nearest distance between sites in different sectors was $4.5 \mathrm{~km}$. Field sites were changed from day to day. Containers were set out in mid-afternoon (1.00-4.00 p.m.) and retrieved the following morning (8.00-11.00 a.m.). 


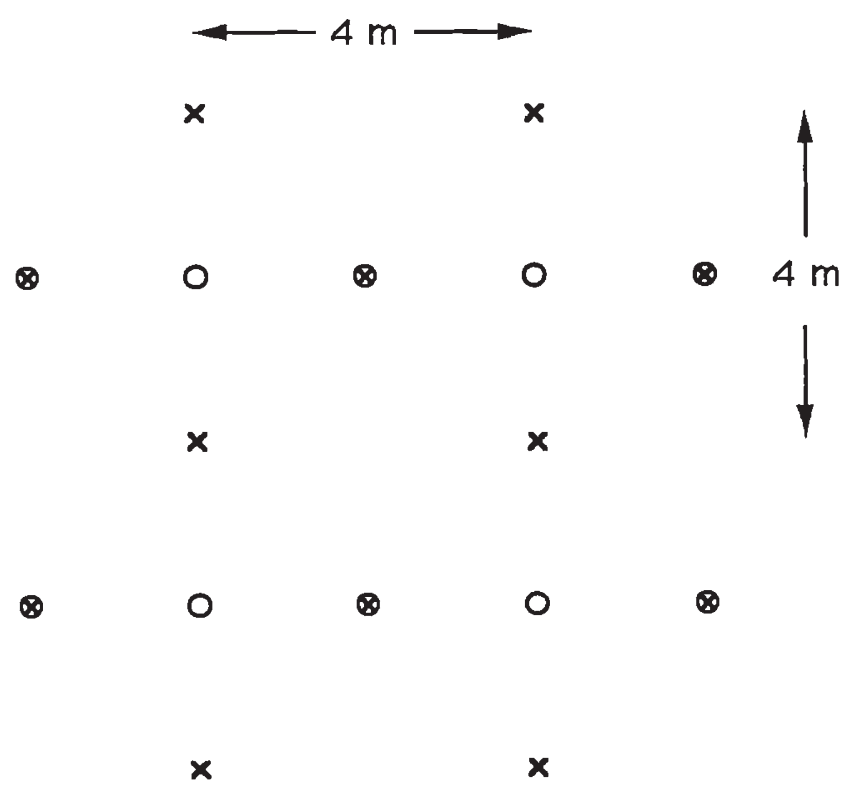

Fig. 2 Layout of containers in the field experiment. $x$ and $\otimes$ mark positions of each of the 12 containers, $\otimes$ marks containers with Pc plates in the one plate/container experiment, o marks positions of each of the four sets of two vials from which flies were released.

For each release, flies had been collected from rearing bottles 7-10 days previously and stored (with transfer every 2-3 days) in fresh yeasted bottles. On the morning of release, approximately 500 pairs per line were collected and stored in eight vials containing 2 per cent agar and then transferred to empty vials just before being taken to the field. At each site, these eight vials were placed at four intermediate spots in the grid (Fig. 2). When retrieved, the number of flies in each container was counted and the number of eggs per plate was counted immediately after returning to the laboratory.

For the experiment with one plate/container, the proportion of eggs laid on Pc-inoculated plates was calculated on a per site basis (i.e. number of eggs laid in the six Pc containers divided by the total number of eggs in all containers). In the experiment with two plates/ container, the proportion of eggs laid on Pc-inoculated plates was calculated on a per container basis and these proportions averaged to obtain the average proportion for the site. Analyses of variance were done on the proportion of eggs laid on Pc plates (both experiments), proportion of adults in Pc containers (one plate/container experiment) and average number of eggs per container (two plates/container experiment). Proportional data were arcsin transformed prior to analysis.

\section{Diallel experiment}

After the field experiment was carried out, the four lines were used in a full diallel experiment. The parental flies for the crosses in this experiment had been maintained for 14 laboratory generations. Experimental procedures were the same as for experiments 1,2 and 4 above and for each of the 16 strains, 10 replicate oviposition chambers were set up, with five females per chamber and two discs each of $P$. cactophila and $C r$. cereanus.

Data on the proportion of eggs laid on Pc in each chamber were analysed according to Method 3 , model I of Griffing (1956), i.e. the lines are a fixed set about which inferences are to be made and parent lines are not included. Analysis using Method 1 (parents included) gave essentially the same results. Data were not transformed for analysis as strain variances were homogeneous and none deviated from normality.

\section{Laboratory retest of selected isofemale lines}

Of the 90 isofemale lines used in experiments 1-4, 40 were selected for retest in the laboratory, using $P$. cactophila and $C r$. cereanus. These 40 lines comprised the 20 that had shown highest preferences for Pc and the 20 with lowest preferences in experiment 2 (which had tested Pc vs. Crc). The lines had been maintained for 31 generations prior to this experiment. Experimental procedures were the same as those for experiments 1,2 and 4 of the isofemale line experiments, except that the yeast species were presented to the flies growing on the surface of a cactus homogenate medium that comprised 30 per cent $O$. stricta homogenate, 1.5 per cent agar (rather than the 10 per cent $O$. stricta homogenate used in experiments 1, 2 and 4), and that 10 replicate oviposition chambers were set up for each isofemale line.

\section{Analysis of yeast volatiles}

The concentrations of volatiles produced by four yeast species ( $\mathrm{Pc}, \mathrm{Crc}$, Clo and another common cactophilic species, Candida sonorensis) when grown in sterilized $O$. stricta homogenate were determined by quantitative gas chromatography. A Varian $3700 \mathrm{GC}$ with flame ionization detectors and a $2 \mathrm{~mm}$ by $2 \mathrm{~mm}$ I.D. by $6.35 \mathrm{~mm}$ O.D. glass column packed with 6.6 per cent Carbowax 20 M on 80/120 Carbopack B AW (Sulpelco, Cat. No. 2-3845) was used. Experiments were carried out in $50 \mathrm{ml}$ flasks which contained $20 \mathrm{ml}$ of 50 per cent sterilized cactus homogenate. Each flask was inoculated with dilute suspensions of the previously mentioned bacterial community and a single yeast species (log phase). Inoculated flasks were incu- 
bated at $25^{\circ} \mathrm{C}$ and samples for analysis were taken at 48 and $72 \mathrm{~h}$. Volatile analysis involved separating the tissue from the liquid by centrifugation in a table-top, clinical centrifuge and injecting 1-5 $\mu \mathrm{l}$ of the supernatant. Peak identification was based on retention time compared with retention times of known compounds and quantification was based on empirically derived regression equations relating peak area and injection of standards of specific concentration.

\section{Results}

\section{Oviposition preferences of isofemale lines}

In experiments 1, 2 and 4, analysis of total eggs per chamber showed no significant differences among populations but highly significant $(P<0.001)$ differences among lines within populations. In experiment 3 , differences among populations were also significant $(P<0.01)$. Mean eggs per chamber in the four experiments, with standard deviations in parentheses, were 212.9 (75.1), 149.9 (87.4), 176.9 (122.1) and 213.7 (86.1), respectively. In experiments 1-3, where females had a choice of two yeast species for oviposition, analyses of variance (Table 1) showed no significant effects when the yeast species were $P$. cactophila and $\mathrm{Cl}$. opuntiae (experiment 1) but highly significant differences among lines within populations $(P<0.001)$ when the yeasts were $P$. cactophila and $C r$. cereanus (experiment 2). Significant differences both among populations $(P<0.001)$ and among lines within populations $(P<0.001)$ were found when the yeasts were $\mathrm{Cr}$. cereanus and $\mathrm{Cl}$. opuntiae (experiment 3 ). The overall means for proportion of eggs laid on $P$. cactophila in experiments 1 and 2 were very similar: $0.6426 \pm 0.1740$ and $0.6418 \pm 0.2246$, respectively, whereas the overall mean proportion on $\mathrm{Cr}$. cereanus in experiment 3 was $0.2973 \pm 0.2286$.

Analyses of variance of the data from experiment 4 were performed in two ways: (i) for the proportion of eggs laid on each of the three yeasts, and (ii) for each of the three pairwise comparisons, ignoring the third yeast (Table 2). There were highly significant differences among lines within populations for all analyses and also significant differences among populations for the proportion of eggs on $\mathrm{Crc}$ and for the $\mathrm{Pc} / \mathrm{Crc}$ comparison. Overall means for the proportions of eggs laid on each yeast were $0.4610 \pm 0.1836 \quad(P$. cactophila $)$, $0.3574 \pm 0.1814$ (Cr. cereanus) and $0.1816 \pm 0.1172$ (Cl. opuntiae).

From each of the ANOVAs, the heritability of individual female preferences can be estimated (Hoffmann \& Parsons, 1988; Barker, 1992). These
Table 1 Analyses of variance of proportions of eggs laid on each yeast in each experiment, the variance component for lines within populations $(V(L / P))$, intra-class correlation $(t)$, heritability of isofemale line means $(H)$ and of individual fly differences $\left(h^{2}\right)$

\begin{tabular}{lrccc}
\hline & \multicolumn{4}{c}{ Mean squares } \\
\cline { 2 - 5 } Source & d.f. & $\begin{array}{c}\text { Exp. 1 } \\
\text { Prop. Pc }\end{array}$ & $\begin{array}{c}\text { Exp. 2 } \\
\text { Prop. Pc }\end{array}$ & \multicolumn{1}{c}{$\begin{array}{c}\text { Exp. 3 } \\
\text { Prop. Crc }\end{array}$} \\
\hline Population $(\mathrm{P})$ & 8 & 0.0416 & 0.1140 & $0.3552^{* * *}$ \\
Lines/P & 81 & 0.0267 & $0.0938^{* * *}$ & $0.0828^{* * *}$ \\
Error & 360 & 0.0308 & 0.0393 & 0.0386 \\
$V(L / P)$ & & Negative & 0.01084 & 0.00908 \\
$t$ & & - & 0.215 & 0.196 \\
Isofemale $H$ & & 0 & 0.052 & 0.046 \\
$h^{2}$ & & 0 & 0.107 & 0.094 \\
\hline
\end{tabular}

a,b Proportions of eggs laid on P. cactophila and Cr. cereanus, respectively.

$* * * P<0.001$.

heritability estimates and the statistics used in calculating them also are given in Tables 1 and 2 .

In all experiments, there was a general trend for the more fecund lines to lay a smaller proportion of eggs on the preferred yeast $(\mathbf{s})$, as shown by the correlation coefficients (Table 3 ). In experiment 4 , Pc was the most preferred yeast and Clo was the least preferred, so that the significant positive correlations between total eggs laid and proportion laid on Clo (Table 3 ) are as meaningful as the negative correlations for the preferred yeasts in experiments $1-3$.

One question of interest in relation to genetic variation for oviposition preferences is whether the rank order of genotypes in their preferences for a particular yeast changes depending on the other yeast species present. Thus, $(i)$ is the rank order of a set of genotypes the same when given the choice of yeasts $i$ and $j$ as for $i$ and $k$, and (ii) is the rank order of preferences for yeast $i$ in a two-way choice changed by the addition of a third yeast into the system? As the same 90 isofemale lines were used in all four experiments, such effects can be detected from ANOVA of the combined data of two experiments. A significant experiment by isofemale line within populations interaction indicates change in rank order of the isofemale lines. Summarized results from these ANOVAs are given in Table 4 for the three comparisons among the two-way choice experiments and for each of these compared with the same yeast combination in the three-way choice experiment. For the two- 
Table 2 Analyses of variance of proportions of eggs laid on each of the three yeasts in experiment 4 and for each of the three pairwise comparisons (ignoring the third yeast), together with the variance component for lines within populations $(V(L / P))$, intra-class correlation $(t)$, heritability of isofemale line means $(H)$ and of individual fly differences $\left(h^{2}\right)$

\begin{tabular}{|c|c|c|c|c|c|c|c|}
\hline Source & d.f. & Prop. Pc & Prop. Crc & Prop. Clo & $\mathrm{Pc} / \mathrm{Crc}$ & $\mathrm{Pc} / \mathrm{Clo}$ & $\mathrm{Crc} / \mathrm{Clo}$ \\
\hline Population $(\mathbf{P})$ & 8 & 0.0928 & $0.1470^{*}$ & 0.0271 & $0.185^{*}$ & 0.057 & 0.147 \\
\hline Lines/P & 81 & $0.0541^{* * *}$ & $0.0606^{* * *}$ & $0.0234^{* * *}$ & $0.075^{* * *}$ & $0.050^{* * *}$ & $0.105^{* * *}$ \\
\hline Error & 360 & 0.0278 & 0.0242 & 0.0113 & 0.033 & 0.030 & 0.038 \\
\hline$t$ & & 0.162 & 0.229 & 0.178 & 0.202 & 0.123 & 0.255 \\
\hline Isofemale $H$ & & 0.037 & 0.056 & 0.042 & 0.048 & 0.027 & 0.064 \\
\hline$h^{2}$ & & 0.076 & 0.115 & 0.085 & 0.098 & 0.055 & 0.132 \\
\hline
\end{tabular}

${ }^{*} P<0.05, * * * P<0.001$.

Table 3 Correlation coefficients between proportions of eggs laid on each yeast and total eggs laid. In experiments $1-3$, correlations are with proportion of eggs laid on the preferred yeasts (Pc in experiments 1 and 2, Clo in experiment 3 ) and in experiment 4 , with proportions on each yeast

\begin{tabular}{lccccc}
\hline & \multicolumn{4}{c}{ Correlation coefficients } \\
\cline { 2 - 6 } & Exp. 1 & Exp. 2 & Exp. 3 & & Exp. 4 \\
\hline Overall & $-0.098^{*}$ & $-0.150^{* *}$ & 0.035 & Pc & -0.017 \\
(448 d.f.) & & & & Crc & -0.056 \\
& & & & Clo & $0.113^{*}$ \\
Within populations & $-0.095^{*}$ & $-0.139^{* *}$ & 0.000 & Pc & -0.044 \\
(441 d.f.) & & & & Crc & -0.025 \\
& & & & Clo & $0.107^{*}$ \\
Within lines/P & -0.078 & -0.095 & -0.104 & Pc & -0.069 \\
(360 d.f.) & & & & Crc & 0.030 \\
& & & & Clo & 0.064 \\
Line means & -0.158 & $-0.219^{*}$ & 0.196 & Pc & 0.060 \\
(88 d.f.) & & & & Crc & -0.166 \\
& & & & Clo & 0.188 \\
\hline$* P<0.05, * * P<0.01$. & & & & &
\end{tabular}

way choice experiments, the interaction is significant in two of the three combined analyses, even though in each case the mean preferences for the yeast common to the two experiments are similar (Pc: experiments 1 and 2, Crc: experiments 2 and 3). The addition of a third yeast to the system also changes preference rankings of isofemale lines for two of the three cases, i.e. addition of Clo to $\mathrm{Pc} / \mathrm{Crc}$ (experiments 2 and 4), and addition of $\mathrm{Pc}$ to $\mathrm{Crc} / \mathrm{Clo}$ (experiments 3 and 4 ).

\section{Selection of lines for the field experiments}

Experiment 2 ( $\mathrm{Pc}$ vs. $\mathrm{Crc}$ ) results were used to select four lines for the field experiment, two with a high preference for oviposition on $P$. cactophila and two with a low preference for this yeast. Of the 90 lines tested, the one with highest preference for Pc (0.9743) was not selected as two of the replicates had only six and eight eggs. The four selected lines were Borah 6 (BOR6), Theodore 9 (T9), Theodore 3 (T3) and 
Mulambin Beach 6 (MB6). Average numbers of eggs and proportions laid on $\mathrm{Pc}$ for these lines are given in Table 5.

The performance of these lines in experiment 4 (Table 5) shows that the two high preference lines (BOR6 and T9) remain above average in preference for Pc whereas the two low lines (T3 and MB6) remain below average. In terms of proportion of eggs laid on $\mathrm{Pc}$, these lines ranked fifth and 25th highest and 11 th and seventh lowest.

\section{Field experiments}

These were carried out during November 1990, with releases on nine days for the two plates/container experiment and on six days for the one plate/container experiment. Table 6 gives the average number of eggs laid and percentage of eggs on Pc-inoculated plates and the average percentage of $D$. buzzatii adults trapped in containers with $\mathrm{Pc}$ plates (as opposed to containers with $\mathrm{Crc}$ plates) in the one plate/container experiment.

In both one plate and two plates/container experiments, only those sites where more than 25 eggs were laid were included in the analyses. For the two plates/ container experiment, date and line were significant for the percentage of eggs laid on Pc (Table 7). For average number of eggs/container, the date effect was significant and the line effect was marginally significant $(P=0.06)$. Percentage eggs laid on Pc was lowest for T3 but the other line selected as having low preference

Table 4 Significance of the experiment $\times$ isofemale lines within populations interaction (indicating change in rank order of isofemale lines) in ANOVA of combined data of two experiments

\begin{tabular}{lcccc}
\hline Experiments & Comparison & $\begin{array}{c}\text { Significance of } \\
\mathrm{E} \times \mathrm{I}(\mathrm{P})\end{array}$ & $\begin{array}{c}\text { Mean proportions } \\
\text { of eggs }\end{array}$ \\
\hline 1 and 2 & $\mathrm{Pc} /$ Clo vs Pc/Crc & $<0.01$ & 0.643 & 0.642 \\
1 and 3 & Clo/Pc vs Clo/Crc & ns & 0.357 & 0.703 \\
2 and 3 & Crc/Pc vs Crc/Clo & $<0.001$ & 0.358 & 0.297 \\
1 and 4 & Pc/Clo & ns & 0.643 & 0.710 \\
2 and 4 & Pc/Crc & $<0.001$ & 0.642 & 0.565 \\
3 and 4 & Crc/Clo & $<0.001$ & 0.297 & 0.647 \\
\hline
\end{tabular}

Mean proportions of eggs in the two-way choice experiments (1,2 and 3$)$ are for the first listed yeast species in each comparison (e.g. proportions of eggs laid on Pc in experiments 1 and 2). For analyses including experiment 4, the proportions are just for the two yeasts given (e.g. for experiments 1 and 4 ( $\mathrm{Pc} / \mathrm{Clo}), 0.710$ is the mean proportion of eggs laid on $\mathrm{Pc}$, relative to $\mathrm{Pc}+\mathrm{Clo}$ only).

Table 5 Average numbers of eggs and proportions laid on Pc for the lines selected for use in the field experiment

\begin{tabular}{|c|c|c|c|c|c|c|c|}
\hline \multirow[b]{3}{*}{ Line } & \multicolumn{2}{|c|}{ Exp. 2} & \multicolumn{5}{|c|}{ Exp. 4} \\
\hline & & & & & & & $\mathrm{Pc}$ \\
\hline & No. of eggs & Prop. Pc & No. of eggs & Prop. Pc & Prop. Crc & Prop. Clo & $\mathrm{Pc}+\mathrm{Crc}$ \\
\hline Borah 6 & $\begin{array}{c}63.0 \\
(58.9)\end{array}$ & $\begin{array}{c}0.9103 \\
(0.1233)\end{array}$ & $\begin{array}{l}137.6 \\
(62.1)\end{array}$ & $\begin{array}{c}0.6359 \\
(0.1050)\end{array}$ & $\begin{array}{c}0.2834 \\
(0.1022)\end{array}$ & $\begin{array}{c}0.0807 \\
(0.0573)\end{array}$ & $\begin{array}{c}0.6919 \\
(0.1053)\end{array}$ \\
\hline Theodore 9 & $\begin{array}{l}120.2 \\
(48.6)\end{array}$ & $\begin{array}{c}0.8925 \\
(0.0610)\end{array}$ & $\begin{array}{l}180.4 \\
(70.0)\end{array}$ & $\begin{array}{c}0.5284 \\
(0.1809)\end{array}$ & $\begin{array}{c}0.3512 \\
(0.1874)\end{array}$ & $\begin{array}{c}0.1204 \\
(0.0239)\end{array}$ & $\begin{array}{c}0.6020 \\
(0.2072)\end{array}$ \\
\hline Theodore 3 & $\begin{array}{l}229.2 \\
(53.8)\end{array}$ & $\begin{array}{c}0.3684 \\
(0.2564)\end{array}$ & $\begin{array}{c}272.2 \\
(43.2)\end{array}$ & $\begin{array}{c}0.3233 \\
(0.0659)\end{array}$ & $\begin{array}{c}0.3579 \\
(0.0657)\end{array}$ & $\begin{array}{c}0.3188 \\
(0.0350)\end{array}$ & $\begin{array}{c}0.4746 \\
(0.0919)\end{array}$ \\
\hline $\begin{array}{l}\text { Mulambin } \\
\text { Beach } 6\end{array}$ & $\begin{array}{c}75.6 \\
(39.8)\end{array}$ & $\begin{array}{c}0.3334 \\
(0.2118)\end{array}$ & $\begin{array}{l}141.0 \\
(91.2)\end{array}$ & $\begin{array}{c}0.3338 \\
(0.2046)\end{array}$ & $\begin{array}{c}0.5103 \\
(0.2389)\end{array}$ & $\begin{array}{c}0.1559 \\
(0.0657)\end{array}$ & $\begin{array}{c}0.4029 \\
(0.2486)\end{array}$ \\
\hline
\end{tabular}

Numbers given are means for five replicates (S.D. in parentheses). 
Table 6 Average numbers of eggs and average percentage laid on Pc plates for each line

\begin{tabular}{|c|c|c|c|c|c|c|c|c|}
\hline \multirow[b]{4}{*}{ Experiment } & \multicolumn{8}{|c|}{ Lines } \\
\hline & \multicolumn{4}{|c|}{ High } & \multicolumn{4}{|c|}{ Low } \\
\hline & \multicolumn{2}{|c|}{ BOR6 } & \multicolumn{2}{|c|}{ T9 } & \multicolumn{2}{|c|}{ T3 } & \multicolumn{2}{|c|}{ MB6 } \\
\hline & Eggs & $\% \mathrm{Pc}$ & Eggs & $\% \mathrm{Pc}$ & Eggs & $\% \mathrm{Pc}$ & Eggs & $\% \mathrm{Pc}$ \\
\hline $\begin{array}{l}\text { Two plates/container } \\
\text { Average/container }\end{array}$ & 30.5 & 97.0 & 42.7 & 93.2 & 42.4 & 76.6 & 45.9 & 91.2 \\
\hline $\begin{array}{l}\text { One plate/container } \\
\text { Average } \\
\text { Average/plate } \\
\text { Average } \% \text { adults in Pc containers }\end{array}$ & 12.1 & $\begin{array}{l}82.0 \\
99.3\end{array}$ & 8.9 & $\begin{array}{r}100.0 \\
99.3\end{array}$ & 7.0 & $\begin{array}{l}97.2 \\
97.3\end{array}$ & 9.4 & $\begin{array}{l}91.1 \\
96.9\end{array}$ \\
\hline
\end{tabular}

Table 7 Two plates/container experiment: analyses of variance of arcsin transformed percentage eggs and mean eggs/container

\begin{tabular}{lrccc}
\hline Source & d.f. & SS & MS & F \\
\hline \multicolumn{5}{l}{ ANOVA: arcsin square root \% Pc eggs } \\
Corrected total & 40 & 2.2708 & - & - \\
Date & 8 & 0.6738 & 0.0842 & $5.63^{* *}$ \\
Line & 3 & 0.7538 & 0.2513 & $16.81^{* * *}$ \\
Date $\times$ line & 15 & 0.4501 & 0.0300 & 2.01 \\
Error & 14 & 0.2093 & 0.0149 & \\
ANOVA: mean eggs per container & & \\
Corrected total & 40 & 48691.6 & - & - \\
Date & 8 & 35271.4 & 4408.9 & $12.77^{* * *}$ \\
Line & 3 & 3281.7 & 1093.9 & $3.17^{*}$ \\
Date $\times$ line & 15 & 5428.2 & 361.9 & 1.05 \\
Error & 14 & 4833.1 & 345.2 & \\
\hline
\end{tabular}

${ }^{*} P=0.06,{ }^{* *} P<0.01,{ }^{* * *} P<0.001$.

for Pc (MB6) showed a percentage of eggs laid on Pc that was not significantly different from the two high preference lines (Tukey's $w$ procedure). Average eggs per container was lowest for BOR6. The only significant effect in the one plate/container experiment was a date effect for percentage adults in Pc containers (Table 8). In this experiment, adults of all lines were settling in the containers with Pc plates, so that there was little opportunity for short-distance choice of oviposition sites. The average number of eggs/container was substantially higher (two to six times) for the two plates/ container experiment than for one plate/container (Table 6). Part of this difference is probably due to temperature effects as the daily maxima were on average higher on those days when the two plates/container
Table 8 One plate/container experiment: analyses of variance of arcsin transformation of both percentage eggs and percentage adults in Pc containers

\begin{tabular}{lrccc}
\hline Source & d.f. & SS & MS & F \\
\hline \multicolumn{5}{l}{ ANOVA: arcsin square root \% Pc eggs } \\
Corrected total & 22 & 1.0154 & - & - \\
Date & 5 & 0.4770 & 0.0954 & 1.90 \\
Line & 3 & 0.0406 & 0.0135 & 0.27 \\
Date $\times$ line & 8 & 0.1357 & 0.0170 & 0.34 \\
Error & 6 & 0.3006 & 0.0501 & - \\
ANOVA: arcsin square root \% Pc adults & & \\
Corrected total & $19^{\text {a }}$ & 0.2432 & - & - \\
Date & 4 & 0.1507 & 0.0377 & $6.40^{*}$ \\
Line & 3 & 0.0336 & 0.0112 & 1.90 \\
Date $\times$ line & 6 & 0.0539 & 0.0090 & 1.53 \\
Error & 6 & 0.0353 & 0.0059 & - \\
\hline
\end{tabular}

${ }^{\text {a }}$ Percentage adults not recorded for three sites on 9

November.

$* P<0.05$.

experiment was carried out. In addition, there may be a synergistic effect in the two plates/container experiment, if females exposed to volatiles from two yeast species are stimulated to lay more eggs.

For each line, the correlation between the average number of flies/container and the average frequency of eggs on $P$. cactophila plates was computed. No correlation was significantly different from zero. For the two plates/container experiment, the correlations between the average total number of eggs laid/container with average frequency of eggs in $P$. cactophila plates were also found to be non-significant compared with zero. 
The original hypothesis was that line preferences documented in the laboratory would be demonstrable under field conditions. We thus categorized the lines according to their laboratory performance. BOR6 and T9 had high preference for P. cactophila whereas MB6 and T3 were low-preference lines. Analysis of the transformed percentage of eggs on Pc-plates according to this categorization also showed significant line effects $(F=7.91$, d.f. $=1,25, P<0.01)$.

\section{Diallel experiment}

Analysis of variance according to Griffing (1956) showed no specific combining ability or reciprocal effects. General combining ability (gca) also was not significant but the gca effects (T9:0.0462, BOR6:0.0411, MB6:-0.0212, T3:-0.0660) rank similarly to the line means in experiment 2 . The exceptions are within each of the high lines and the low lines where, in both cases, the rankings are reversed in the diallel compared with experiment 2. Comparing gca effects using the standard error of the difference $(0.0526)$, the highest line (T9) is significantly greater than the lowest (T3) $(t=2.13, P<0.05)$.

\section{Laboratory retest of selected isofemale lines}

The product-moment correlation coefficient between the mean (over replicates) proportion of eggs laid on Pc by each line in experiment 2 and in the retest was 0.388 $(P<0.05)$ whereas the Spearman rank correlation coefficient was $0.308(P<0.05)$. The means for each line in the two experiments were on average substantially higher in the retest $(0.8735$ vs. 0.6399$)$. For the four lines used in the field experiment, the two high Pc lines (BOR6 and T9) ranked second and third in experiment 2 and 14th and fifth in the retest, both still higher than the high Pc mean. The two low Pc lines (MB6 and T3) which ranked lowest and second lowest in experiment 2 retained these same rankings in the retest.

\section{Analysis of yeast volatiles}

The concentrations of the major volatiles detected in 50 per cent $O$. stricta tissue homogenate $48 \mathrm{~h}$ after being inoculated with cactophilic yeasts are presented in Table 9. In most cases, all concentrations were slightly reduced at $72 \mathrm{~h}$ compared with $48 \mathrm{~h}$ and consequently only the latter data are given. The major difference between the yeast species involves the production of ethanol. Two of the yeasts, $C$. sonorensis and $\mathrm{Cl}$. opuntiae, are fermenters producing high concentrations of this alcohol. The other two species ( $P$. cactophila and $C r$. cereanus) produce less than 10 per cent
Table 9 Concentrations (mm) of volatiles produced by cactophilic yeasts on Opuntia stricta tissue after $48 \mathrm{~h}$

\begin{tabular}{lcccc}
\hline & \multicolumn{4}{c}{ Yeast species } \\
\cline { 2 - 5 } Volatile & Cs & Pc & Clo & Crc \\
\hline Acetaldehyde & 0.8 & 0.6 & 0.9 & 0.8 \\
Methanol & 2.1 & 2.4 & 2.3 & 2.5 \\
Ethanol & 85.5 & 7.1 & 97.9 & 2.7 \\
Iso-Butanol & 0.01 & 0 & 0.01 & 0 \\
Acetic acid & 4.9 & 4.4 & 3.2 & 4.1 \\
Propionic acid & 3.3 & 4.7 & 1.2 & 4.5 \\
Iso-Butyric acid & 0 & 0.4 & 0 & 0.4 \\
$n$-Butyric acid & 7.4 & 10.0 & 2.1 & 5.2 \\
\hline
\end{tabular}

Cs: Candida sonorensis; Pc: Pichia cactophila; Clo:

Clavispora opuntiae; Crc: Cryptococcus cereanus.

of the concentration produced by the fermenters. One additional difference between the species is that $\mathrm{Cl}$. opuntiae appears to produce less propionic and $n$ butyric acids than the other three yeasts.

\section{Discussion}

Many of the previous studies of genetic variation for habitat preference in Drosophila species relate to broadly defined habitats (Klaczko et al., 1986) or to different host plants or substrates (fruits) of different species (Carson \& Ohta, 1981; Hoffmann et al., 1984; Jaenike, 1985; Lofdahl, 1986; Courtney \& Chen, 1988). There is a spatial hierarchy in habitat choice, where flies may move to habitats or host plants that are generally suitable and then choose microhabitats for oviposition. This distinction has been emphasized by Jaenike (1986) in defining settling behaviour as movement to and settling on a potential host, followed by oviposition site preference. Genetic variation for both these behaviours was found for $D$. tripunctata by Jaenike (1986) but his results indicated them to be independent genetically.

The isofemale line experiments specifically simulate oviposition site preferences within rots. Natural rots contain one to five yeast species (Barker et al., 1983, 1984) but the yeast species distribution within rots is patchy (Barker et al., 1987). In the field experiments, the two plates/container experiment also simulates this within-rot oviposition choice. With only one plate/ container, however, settling behaviour in relation to either Pc or Crc apparently predominated, with most adults of all four lines being attracted to Pc, and consequently, short-distance oviposition site preferences were not expressed. 
The primary rationale of the field experiment was to test whether laboratory expressed-oviposition site preferences would be repeatable under simulated natural conditions. Clearly the one plate/container experiment provided no information. In the two plates/ container experiment, all four lines showed a higher preference for $\mathrm{Pc}$ than in the laboratory and one selected on low laboratory Pc preference (MB6) did not differ significantly from the two high Pc lines (BOR6, T9). Thus laboratory preferences were in general repeatable under simulated natural conditions but the result for line MB6 raised the question whether its preferences had changed during the nine generations of laboratory maintenance between experiment 2 and the field experiment. Results of the diallel experiment (carried out two generations after the field experiment) are not definitive. Although MB6 did not differ significantly from the two high Pc lines for gca effects, the power of this test is very low. The laboratory retest of selected isofemale lines, however, clearly demonstrated that MB6 had not changed: it again showed the lowest preference for Pc, after a further 18 generations of laboratory maintenance following the field experiment. The higher preference for Pc expressed by line MB6 in the field experiment apparently was some function of the conditions of that experiment, possibly relating to settling behaviour, which is not expressed in the small, closed oviposition chambers in the laboratory.

All four lines in the field experiment, however, and the 40 lines in the retest experiment had higher preferences for Pc compared with experiment 2. The only difference in procedures was the substrate used for yeast growth: 30 per cent $O$. stricta for the field experiment and the retest and 10 per cent for experiment 2 . Thus 20 lines were again tested, using the two substrates as treatments. Average proportions of eggs laid on Pc were significantly different $(P<0.001 ; 10$ per cent $=0.615,30$ per cent $=0.752$ ) and the treatment $\times$ line interaction was not significant. The 30 per cent $O$. stricta substrate produces better yeast growth and under these conditions $P$. cactophila is more attractive, presumably because of increased volatile production (see below).

The results of the isofemale line experiments support and extend those of Barker (1992), where females were given a choice of five yeast species including those used here. In those experiments, significant genetic variation was detected and the heritabilities of individual female preferences for each yeast (i.e. proportions of eggs laid on yeast $i$ vs. the other four) were generally not significantly different from zero for Candida sonorensis and $\mathrm{Cl}$. opuntiae but were significant and ranged up to about 10 per cent for $P$. cacto- phila, Cr. cereanus and Candida mucilagina. When females were given a choice between two yeast species in the present experiments (Table 1), significant genetic variation was found for the choice between $P$. cactophila and Cr. cereanus (experiment 2) and for that between $\mathrm{Cr}$. cereanus and $\mathrm{Cl}$. opuntiae (experiment 3) but not for $\mathrm{P}$. cactophila and $\mathrm{Cl}$. opuntiae (experiment 1). When females were given a choice among all three yeast species, the heritability of individual female preferences for each yeast was significant (Table 2). In this case, interactions among the yeasts apparently affect oviposition preferences and the proportions of eggs laid on each yeast are not directly predictable from the pairwise preferences (Table 4).

The significant genetic variation among isofemale lines for oviposition site preferences is expressed in terms of the choice of $C r$. cereanus, with heritability significantly different from zero for both two-way yeast choice experiments that included $\mathrm{Crc}$ (experiments 2 and 3 , Table 1 ). In experiment 4 , the heritability of the proportion of eggs laid on $\mathrm{Cr}$. cereanus is higher than for the other two yeasts (Table 2).

Interactions among the yeast species affecting female preferences would broaden the scope for habitat selection. The preference rankings of the isofemale lines for $\mathrm{Pc}$ in experiment 2 and for $\mathrm{Crc}$ in experiment 3 were both changed by the addition of a third yeast species (Clo and Pc, respectively; Table 4). Thus in natural populations, it will not be simply that some yeasts are preferred by some genotypes and other yeasts by other genotypes but that the preferences themselves may vary depending on each particular combination of yeast species present in a rot.

The absence of genetic variation for preferences for C. sonorensis and $\mathrm{Cl}$. opuntiae as oviposition sites may be due to their fermentation ability and volatile production. Both species are fermenters that produce similar concentrations of volatiles when grown on $O$. stricta tissue (Table 9), with particularly high concentrations of ethanol. Genetic differences thus may not be expressed as variation in oviposition site preferences if D. buzzatii females in general are attracted to ethanol. The volatiles produced by the microorganisms present in cactus rots most probably provide the cues to flies seeking feeding or oviposition sites (Fogleman, 1982; Fogleman \& Abril, 1990). Responses of flies to these volatiles occur at two levels: (i) long-distance responses to different rots by flies in flight, and (ii) short-distance (walking) response within a rot (Barker, 1992). The responses of $D$. buzzatii to five volatiles have been tested by Armstrong (1992), using both a Y-tube olfactometer (short-distance walking response) and a wind tunnel (flying response), comparing each volatile with a water control. Forty isofemale lines were 
tested in the Y-tube and the mean responses to ethanol and methanol were not significantly different but both were significantly less than the response to iso-butanol, iso-amyl alcohol and ethyl acetate. Four of these lines, selected on the diversity of their responses in the Y-tube, then were tested in the wind tunnel, where ethanol was on average the most attractive. Spearman rank correlations for each line between responses in the two olfactometers were not significant for three lines but were significant for the one line which showed highest attraction to ethanol in both tests. Thus, ethanol produced by $\mathrm{C}$. sonorensis and $\mathrm{Cl}$. opuntiae may well be a major component affecting long-distance attraction to rots but with the long-distance and shortdistance responses being genetically independent.

\section{Acknowledgements}

This research was supported by a NSF grant (INT8913365 ) to J.C.F. and W.T.S. and an Australian Research Council grant to J.S.F.B. We are indebted to Don Fredline, Annette Edmonds, Shari Henderson, Margaret Low, Bob Krebs and Michelle Lincoln for laboratory assistance and to Eric Toolson and Tim Armstrong for their assistance with the field experiment.

\section{References}

ARMSTRONG, T. P. 1992. Olfaction and habitat selection in Drosophila buzzatii. Ph.D Thesis, University of New England, Armidale.

BARKER, J. S. F. 1992. Genetic variation in cactophilic Drosophila for oviposition on natural yeast substrates. Evolution, 46, 1070-1083.

BARKER, J. S. F., EAST, P. D., PHAFF, H. J. AND MIRANDA, M. 1984. The ecology of the yeast flora in necrotic Opuntia cacti and of associated Drosophila in Australia. Microbial Ecol., 10, 379-399.

BARKER, J. S. F., STARMER, W. T. AND VACEK, D. C. 1987. Analysis of spatial and temporal variation in the community structure of yeasts associated with decaying Opuntia cactus. Microbial Ecol., 14, 267-276.

BARKER, J. S. F., TOLL, G. L., EAST, P. D., MIRANDA, M. AND PHAFF, H. J. 1983. Heterogeneity of the yeast flora in the breeding sites of cactophilic Drosophila. Can. J. Microbiol., 29, 6-14.

CARSON, H. L. AND OHTA, A. T. 1981. Origin of the genetic basis of colonizing ability. In: Scudder, G. G. E. and Reveal, J. L. (eds) Evolution Today, pp. 365-370. Carnegie-Mellon University, Pittsburgh.

COURTNEY, S. P. AND CHEN, G. K. 1988. Genetic and environmental variation in oviposition behavior in the mycophagous Drosophila suboccidentalis Spcr. Funct. Ecol., 2, 521-528.

DIEHL, S. R. AND BUSH, G. L. 1989. The role of habitat preference in adaptation and speciation. In: Ott, D. and Endler, J. A. (eds) Speciation and its Consequences, pp. 345-365. Sinauer, Sunderland, MA.

FELLOws, D. P. AND HEED, w. B. 1972. Factors affecting host plant selection in desert-adapted cactiphilic Drosophila. Ecology, 53, 850-858.

FOGLEMAN, J. C. 1982. The role of volatiles in the ecology of cactophilic Drosophila. In: Barker, J. S. F. and Starmer, W. T. (eds) Ecological Genetics and Evolution. The Cactus-Yeast-Drosophila Model System, pp. 191-206. Academic Press Australia, Sydney.

FOGLEMAN, J. C. AND ABRiL, J. R. 1990. Ecological and evolutionary importance of host plant chemistry. In: Barker, J. S. F., Starmer, W. T. and MacIntyre, R. J. (eds) Ecological and Evolutionary Genetics of Drosophila, pp. 121-143. Plenum, New York.

GARCIA-DORADO, A. 1986. The effect of niche preference on polymorphism protection in a heterogeneous environment. Evolution, 40, 936-945.

GARCIA-DORADO, A. 1987. Polymorphism from environmental heterogeneity: some features of genetically induced niche preference. Theor. Pop. Biol., 32, 66-75.

GRIFFING, B. 1956. Concept of general and specific combining ability in relation to diallel crossing systems. Aust. J. Biol. Sci., 9, 463-493.

HEDRICK, P. W. 1990a. Genotypic-specific habitat selection: a new model and its application. Heredity, 65, 145-149.

HEDRICK, P. w. 1990b. Theoretical analysis of habitat selection and the maintenance of genetic variation. In: Barker, J. S. F., Starmer, W. T. and MacIntyre, R. J. (eds) Ecological and Evolutionary Genetics of Drosophila, pp. 209-227. Plenum, New York.

HEED, w. B. 1971. Host plant specificity and speciation in Hawaiian Drosophila. Taxon, 20, 115-121.

HOFFMANN, A. A. AND PARSONS, P. A. 1988. The analysis of quantitative variation in natural populations with isofemale strains. Génét. Sél. Évol., 20, 87-98.

HOFFMANN, A. A., PARSONS, P. A. AND NIELSEN, K. M. 1984. Habitat selection: olfactory response of Drosophila melanogaster depends on resources. Heredity, 53, 139-143.

JAENIKE, J. 1985. Genetic and environmental determinants of food preference in Drosophila tripunctata. Evolution, 39, 362-369.

JAENiKe, J. 1986. Genetic complexity of host-selection behavior in Drosophila. Proc. Natl. Acad. Sci. U.S.A., 83, 2148-2151.

JAENIKE, J. AND HOLT, R. D. 1991. Genetic variation for habitat preference: evidence and explanations. Am. Nat., 137, S67-S90.

KLACZKo, L. B., TAYLOR, C. E. AND POWELl, J. R. 1986. Genetic variation for dispersal by Drosophila pseudoobscura and Drosophila persimilis. Genetics, 112, 229-235.

LOFDAHL, K. L. 1986. A genetic analysis of habitat selection in the cactophilic species, Drosophila mojavensis. Huettel, M. D. (ed.) Evolutionary Genetics of Invertebrate Behavior, pp. 153-162. Plenum, New York.

MAYNARD SMITH, J. 1970. Genetic polymorphism in a varied environment. Am. Nat., 104, 487-490.

ORIANS, G. H. (ED.) 1991. Habitat selection. Am. Nat., 137, S1-S130. 
RAUSHEK, M. D. 1984. The evolution of habitat preference in subdivided populations. Evolution, 38, 596-608.

ROSENZWEIG, M. L. (ED.) 1987. Habitat selection and evolutionary processes. Evol. Ecol., 1, 283-407.

SOKAL, R. R. AND ROHLF, F. J. 1981, Biometry. W. H. Freeman \& Co., San Francisco.

STARMER, w. T. AND BARKER, J. S. F. 1986. Ecological genetics of the Adh-1 locus of Drosophila buzzatii. Biol. J. Linn. Soc., 28, 373-385.

TAYLOR, C. E. 1976. Genetic variation in heterogeneous environments. Genetics, 83, 887-894.

TEMPLETON, A. R. AND ROTHMAN, E. D. 1981. Evolution in finegrained environments. II. Habitat selection as a homeostatic mechanism. Theor. Pop. Biol., 19, 326-340. 